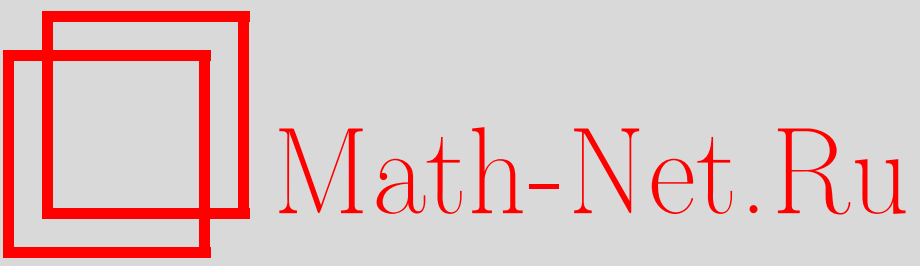

Г. С. Шаров, Аналоги рядов Фурье для модели релятивистской струны с массивными концами, ТМФ, 1996, том 107, номер 1, 86-99

DOI: https://doi.org/10.4213/tmf1140

Использование Общероссийского математического портала Math-Net.Ru подразумевает, что вы прочитали и согласны с пользовательским соглашением

http://www.mathnet.ru/rus/agreement

Параметры загрузки:

IP : 54.237 .206 .68

26 апреля 2023 г., $17: 43: 24$ 
ТЕОРЕТИЧЕСКАЯ

И МАТЕМАТИЧЕСКАЯ

ФИЗИКА

Том 107, № 1

апрель, 1996

\author{
Г. С. Шаров
}

\title{
АНАЛОГИ РЯДОВ ФУРЬЕ ДЛЯ МОДЕЛИ РЕЛЯТИВИСТСКОЙ СТРУНЫ С МАССИВНЫМИ КОНЦАМИ
}

\begin{abstract}
Предложено описание движений релятивистской струны с массивными концами (на примере модели с конечной массой первого конца и бесконечной - второго), основанное на том, что мировая поверхность струны представима в виде ряда, который из-за нелинейности задачи не сводится к обычному ряду Фурье. Уравнение состояния струны следует из условия массовой поверхности для ее конца. Классифициированы движения рассматриваемой струны, допускающие с помощью натуральной параметризации траектории ее подвижного конца линеаризацию соответствующего краевого условия. Показана ограниченность множества таких мировых поверхностей, в частности, в 2+1и 3+1-мерных пространствах Минковского все они сводятся к геликоиду.
\end{abstract}

\section{ВВЕДЕНИЕ}

Модель релятивистской струны с массами на концах [1-6], используемая для описания связанной сильным взаимодействием системы из двух кварков, послужила одним из источников бурного развития ныне весьма разветвленной теории струн (см. обзор [7]). Однако прогресс в теории струны с массивными концами сдерживается сушественным препятствием, возникаюшим при переходе к квантовому описанию этой модели вследствие ее нелинейности.

Суть проблемы в том, что теория струн развивается как двумерная теория поля, в которой это поле удовлетворяет при наличии некоторой связи уравнению колебаний струны - двумерному аналогу волнового уравнения [1-8]. В моделях замкнутой и открытой свободной (с безмассовыми концами) струн данное поле, представляюшее собой координату точки струны $X^{\mu}$, подчинено линейным краевым условиям, которые позволяют разложить поле по двумерным плоским волнам. Математически это эквивалентно разложению в ряд Фурье периодических функций одного аргумента. При квантовании амплитуды гармоник этого ряда отождествляются с квантовыми операторами.

В модели струны с массивными концами краевые условия имеют сушественно нелинейный вид [1-4]. Следовательно, сумма двух удовлетворяюших им функций, вообще говоря, не будет удовлетворять этим условиям. Противоречие квантовому принципу суперпозиции не позволяет применить здесь упомянутую выше схему квантования, основанную на разложении $X^{\mu}$ в ряд.

В настояшей работе введены некоторые аналоги рядов Фурье, в виде которых можно представить мировую поверхность $X^{\mu}$, определяемую нелинейными краевыми условия- 
ми. В первом разделе приводятся основные уравнения, описьвающие рассматриваемую модель, а также некоторые соотношения, полученные в работах [5,9], которые касаются единичного вектора скорости конца струны. Эта величина, с точностью до транслящий определяющая мировую поверхность струны, играет важную роль в предлагаемом подходе. Последний изложен в разделе 2 на примере струны с бесконечно тяжелым (фиксированным) вторым концом. Эта модель описывает, в частности, и центрально-симметричные движения струны с одинаковыми массами на концах.

В разделе 3 исследуется класс движений данной струны, которые допускают натуральную параметризацию траектории ее подвижного конца.

\section{1. УРАВНЕНИЯ ДВИЖЕНИЯ И КРАЕВЫЕ УСЛОВИЯ ДЛЯ РЕЛЯТИВИСТСКОЙ СТРУНЫ}

Движение релятивистской струны с натяжением $\gamma$ и массами $m_{1}, m_{2}$ на концах описывается действием [1-4]

$$
S=-\int_{\tau_{1}}^{\tau_{2}} d \tau\left\{\gamma \int_{\sigma_{1}(\tau)}^{\sigma_{2}(\tau)}\left[\left(\dot{X} X^{\prime}\right)^{2}-\dot{X}^{2} X^{\prime 2}\right]^{\frac{1}{2}} d \sigma+\sum_{a=1}^{2} m_{a} \sqrt{\left[\frac{d}{d \tau} X^{\mu}\left(\tau, \sigma_{a}(\tau)\right)\right]^{2}}\right\}
$$

Здесь $X^{\mu}(\tau, \sigma)$ - координаты точки струны в $D$-мерном пространстве Минковского $\mathbb{R}^{1, D-1}, \sigma_{1}(\tau)$ и $\sigma_{2}(\tau)$ - внутренние координаты концов струны, $\dot{X}^{\mu}=\partial X^{\mu} / \partial \tau, X^{\prime \mu}=$ $\partial X^{\mu} / \partial \sigma ;(a b)=a^{\mu} b_{\mu}=\eta_{\mu \nu} a^{\mu} b^{\nu}-($ псевдо) $)$ скалярное произведение в $\mathbb{R}^{1, D-1}$, скорость света $c=1$.

Варьирование действия (1.1) дает уравнения движения струны и граничные условия, которые принимают простейший вид, если выбором координат $\tau$ и $\sigma$ сделать индуцированную метрику на мировой поверхности струны конформно-плоской, т.е. удовлетворяюшей условиям ортонормальной калибровки

$$
\dot{X}^{2}+X^{\prime 2}=0, \quad\left(\dot{X} X^{\prime}\right)=0
$$

При выполнении этих условий уравнения движения становятся линейными:

$$
\ddot{X}^{\mu}-X^{\prime \prime \mu}=0
$$

а краевые условия на нумеруемых индексом $a$ концах струны принимают вид

$$
\left.\left\{m_{a} \frac{d}{d \tau} \frac{\dot{X}^{\mu}+\sigma_{a}^{\prime}(\tau) X^{\prime \mu}}{\sqrt{\dot{X}^{2} \cdot\left(1-\sigma_{a}^{\prime 2}\right)}}+(-1)^{a} \gamma\left[X^{\prime \mu}+\sigma_{a}^{\prime}(\tau) \dot{X}^{\mu}\right]\right\}\right|_{\sigma=\sigma_{a}(\tau)}=0
$$

Преобразуем краевые условия (1.4), подставив в них общее решение (1.3),

$$
X^{\mu}(\tau, \sigma)=\frac{1}{2}\left[\Psi_{+}^{\mu}(\tau+\sigma)+\Psi_{-}^{\mu}(\tau-\sigma)\right] .
$$

Производные введенных здесь функций вследствие условий (1.2) изотропны:

$$
\Psi_{-}^{\prime 2}(\xi)=\Psi_{+}^{\prime 2}(\xi)=0
$$


Зафиксируем внутренние уравнения траекторий концов струны в виде

$$
\sigma_{1}(\tau)=0, \quad \sigma_{2}(\tau)=\pi
$$

что всегда можно сделать $[1,4]$, используя инвариантность выражений $(1.2)$ и $(1.3)$ относительно преобразований

$$
\tilde{\tau} \pm \tilde{\sigma}=f_{ \pm}(\tau \pm \sigma) .
$$

Отметим, что условия (1.7) фиксируют свободу выбора функций $f_{ \pm}(\xi)$ не окончательно. Если $\phi(\xi)$ - произвольная $2 \pi$-периодическая гладкая функция с ограниченной производной, то замены переменных класса (1.8) с

$$
f_{+}(\xi)=f_{-}(\xi)=\xi+\phi(\xi), \quad \phi(\xi+2 \pi)=\phi(\xi), \quad\left|\phi^{\prime}(\xi)\right|<1
$$

сохраняют вид (1.7) уравнений конщов струны: $\tilde{\sigma}=0$ и $\tilde{\sigma}=\pi$. Произвол в выборе координат (1.9) на мировой поверхности (1.5) можно интерпретировать как физическую несушественность “продольных колебаний” внутренних точек струны (в рамках действия (1.1) эти точки неразличимы).

После подстановки (1.5) в (1.4), учета (1.7) и интегрирования краевые условия для конечных ненулевых масс на концах струны примут вид

$$
\frac{\Psi_{+}^{\prime \mu}\left(\tau+\sigma_{a}\right)+\Psi_{-}^{\prime \mu}\left(\tau-\sigma_{a}\right)}{\sqrt{2\left(\Psi_{+}^{\prime}\left(\tau+\sigma_{a}\right) \Psi_{-}^{\prime}\left(\tau-\sigma_{a}\right)\right)}}+\frac{(-1)^{a} \gamma}{2 m_{a}}\left[\Psi_{+}^{\mu}\left(\tau+\sigma_{a}\right)-\Psi_{-}^{\mu}\left(\tau-\sigma_{a}\right)\right]=W_{a}^{\mu} .
$$

Здесь $W_{a}^{\mu}$ - постоянные интегрирования. Введем в качестве новых переменных единичные векторы скорости концов струны $\left(U^{2}=V^{2}=1\right)$ :

$$
\begin{aligned}
& U^{\mu}(\tau)=\frac{\dot{X}^{\mu}(\tau, 0)}{\sqrt{\dot{X}^{2}(\tau, 0)}}=\frac{\Psi_{+}^{\prime \mu}(\tau)+\Psi_{-}^{\prime \mu}(\tau)}{\sqrt{2\left(\Psi_{+}^{\prime}(\tau) \Psi_{-}^{\prime}(\tau)\right)}} \\
& V^{\mu}(\tau)=\frac{\dot{X}^{\mu}(\tau, \pi)}{\sqrt{\dot{X}^{2}(\tau, \pi)}}=\frac{\Psi_{+}^{\prime \mu}(\tau+\pi)+\Psi_{-}^{\prime \mu}(\tau-\pi)}{\sqrt{2\left(\Psi_{+}^{\prime}(\tau+\pi) \Psi_{-}^{\prime}(\tau-\pi)\right)}} .
\end{aligned}
$$

Домножая скалярно $(1.11)$ на $\Psi_{+}^{\prime \mu}(\tau)$, получаем с учетом $(1.6)$ выражение $\sqrt{2\left(\Psi_{+}^{\prime} \Psi_{-}^{\prime}\right)}=$ $2\left(U \Psi_{+}^{\prime}\right)$, которое после подстановки его в (1.11) позволяет преобразовать (1.10) к нормальной (разрешенной относительно производных) системе обыкновенных дифференциальных уравнений [5]

$$
\frac{d U^{\mu}}{d \tau}=k_{1}\left(\eta^{\mu \nu}-U^{\mu} U^{\nu}\right) \Psi_{+\nu}^{\prime}(\tau)
$$

Здесь $\eta^{\mu \nu}=\operatorname{diag}(1 ;-1 ;-1 ; \ldots), \eta^{\mu \nu} \eta_{\nu \lambda}=\delta_{\lambda}^{\mu}$, а также введено обозначение $k_{1}=\gamma / m_{1}$ для постоянной кривизны траектории конца струны [3].

Интегрируя систему (1.13) и пользуясь следуюшим из (1.10) соотношением

$$
U^{\mu}(\tau)=W_{1}^{\mu}+\frac{\gamma}{2 m_{1}}\left[\Psi_{+}^{\mu}(\tau)-\Psi_{-}^{\mu}(\tau)\right]
$$


мы можем по известной на некотором отрезке функции $\Psi_{+}^{\mu}(\tau)$ однозначно определить на этом отрезке функцию $\Psi_{-}^{\mu}(\tau)$ (постоянные интегрирования фиксируются начальным условием $U^{\mu}(0)$ на данном конце струны [5]).

Краевое условие для другого конца позволяет установить связь между $\Psi_{+}^{\mu}(\tau+\pi)$ и $\Psi_{-}^{\mu}(\tau-\pi)$, которая в случае безмассового второго конца имеет простейший вид, следуюший из (1.4) или (1.10):

$$
\Psi_{+}^{\prime \mu}(\tau+\pi)=\Psi_{-}^{\prime \mu}(\tau-\pi), \quad m_{2}=0
$$

Если масса второго конца отлична от нуля и конечна, то по аналогии с (1.13) - (1.14) эта связь осушествляется соотношениями

$$
\begin{aligned}
\frac{d V^{\mu}}{d \tau} & =\frac{\gamma}{m_{2}}\left[\delta_{\nu}^{\mu}-V^{\mu}(\tau) V_{\nu}(\tau)\right] \Psi_{-}^{\prime \nu}(\tau-\pi) \\
V^{\mu}(\tau) & =W_{2}^{\mu}-\frac{\gamma}{2 m_{2}}\left[\Psi_{+}^{\mu}(\tau+\pi)-\Psi_{-}^{\mu}(\tau-\pi)\right] .
\end{aligned}
$$

В предельном случае бесконечно тяжелого второго конца $\left(m_{2} \rightarrow \infty\right)$ краевое условие (1.10) примет вид

$$
V^{\mu}(\tau)=W_{2}^{\mu} \equiv W^{\mu}
$$

Это значит, что бесконечно тяжелый конец, не отклоняемый струной, движется прямолинейно с постоянной скоростью, описываемой единичным $\mathbb{R}^{1, D-1}$-вектором $W^{\mu}$. Всегда можно выбрать систему отсчета, в которой данньй конец покоится. В этом смысле будем считать конец с $m \rightarrow \infty$ фиксированным.

Домножая условие $(1.18)$ с $V^{\mu}(\tau)$ в виде (1.12) скалярно на $\Psi_{+}^{\prime \mu}(\tau+\pi)$ или $\Psi_{-}^{\prime \mu}(\tau-\pi)$, получим следуюшие соотношения, связывающие $\Psi_{+}^{\prime \mu}$ и $\Psi_{-}^{\prime \mu}[5]$ :

$$
\Psi_{ \pm}^{\prime \mu}(\xi \pm 2 \pi)=\left(2 W^{\mu} W_{\nu}-\delta_{\nu}^{\mu}\right) \Psi_{\mp}^{\prime \nu}(\xi)
$$

В работе [9] показано, что мировая поверхность струны однозначно восстанавливается по заданной траектории ее конца. Этот вывод базируется на однозначной разрешимости системы (1.13) относительно $\Psi_{+}^{\prime \mu}(\tau)$. Возводя в скалярный квадрат обе части (1.13), учитывая при этом (1.6) и единичность $U^{\mu}$, получим соотношение $U^{\prime} 2(\tau)=$ $-k_{1}^{2}\left(U \Psi_{+}^{\prime}\right)^{2}$. После извлечения квадратного корня с учетом ориентации $U^{\mu}$ и $\Psi_{+}^{\prime \mu}$ в конус будущего: $\left(U \Psi_{+}^{\prime}\right)=k_{1}^{-1} \sqrt{-U^{\prime 2}}$, и подстановки его в (1.13) мы придем к следующему выражению для $\Psi_{+}^{\prime \mu}$, а также с помощью $(1.14)$ - для $\Psi_{-}^{\prime \mu}[9]$ :

$$
\Psi_{ \pm}^{\prime \mu}(\tau)=\frac{1}{k_{1}}\left[ \pm U^{\prime \mu}(\tau)+\sqrt{-U^{\prime 2}(\tau)} U^{\mu}(\tau)\right]
$$

Эти формулы позволяют восстановить мировую поверхность струны по заданной траектории ее конца или, что эквивалентно, - по заданным $k_{1}$ и $U^{\mu}(\tau)$.

В том случае, когда мы обладаем некоторой информацией о движении второго конца, для определения мировой поверхности достаточно задать вектор-функцию $U^{\mu}(\tau)$ (или $\left.V^{\mu}(\tau)\right)$ на конечном отрезке. Данный отрезок ограничен "моментом" $\tau_{0}$ отправления сигнала со световой скоростью от конца вдоль струны и "моментом" $\tau_{2}$ возврашения этого сигнала к исходному концу после отражения (соответствуюшего $\tau_{1}$ ) от другого 
конца. Мировые линии этих сигналов - характеристики $\tau \pm \sigma=$ const. Информация о втором конце должна включать в себя сведения о его массе $m_{2}$ или кривизне траектории $k_{2}$ и о единичном векторе скорости в точке отражения $V^{\mu}\left(\tau_{1}\right)$.

При обосновании данного утверждения ограничимся случаем (1.7), при этом упомянутый отрезок имеет длину $\tau_{2}-\tau_{0}=2 \pi$. Пусть $0<m_{2}<\infty$. Предположив, что

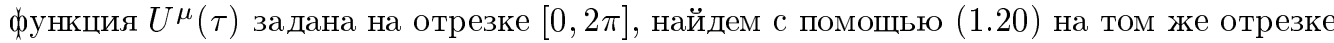
$\Psi_{ \pm}^{\prime \mu}(\tau)$. После подстановки $\Psi_{-}^{\prime \mu}$ в систему $(1.16)$ и ее интегрирования можно получить вектор-функцию $V^{\mu}(\tau)$ на отрезке $\tau \in[\pi, 3 \pi]$, зависящую от начального условия для системы - постоянного вектора $V^{\mu}(\pi)=W^{\mu}$. После этого с помошью соотношения $(1.17)$ находим $\Psi_{+}^{\mu}(\tau)$ на отрезке $[2 \pi, 4 \pi]$, а затем, подставив $\Psi_{+}^{\mu}$ в $(1.13)$, продолжаем на этот отрезок $U^{\mu}(\tau)$. Процедура продолжения $U^{\mu}$ и $V^{\mu}$ может повторяться рекуррентHо.

Описанный механизм упрощается для случая бесконечно тяжелого второго конца $m_{2} \rightarrow \infty$ (в настояшей работе в центре внимания находится именно эта модель). Подставляя выражения (1.19) и (1.20) в (1.13), получим систему обыкновенных дифференциальных уравнений

$$
\begin{aligned}
U^{\prime \mu}(\tau+2 \pi)= & {\left[\delta_{\nu}^{\mu}-U^{\mu}(\tau+2 \pi) U_{\nu}(\tau+2 \pi)\right]\left(2 W^{\nu} W_{\lambda}-\delta_{\lambda}^{\nu}\right) \times } \\
& \times\left[-U^{\prime \lambda}(\tau)+\sqrt{-U^{\prime 2}(\tau)} U^{\lambda}(\tau)\right]
\end{aligned}
$$

позволяющую неограниченно продолжать $U^{\mu}(\tau)$, если эта функция задана на отрезке $[0,2 \pi]$, а также известна скорость второго конца $W^{\mu}$.

Не представляет труда, используя (1.15), написать аналогичную систему и для случая $m_{2}=0$, заменив в $(1.21)$ матрицу $\left(2 W^{\nu} W_{\lambda}-\delta_{\lambda}^{\nu}\right)$ на $\delta_{\lambda}^{\nu}$.

Отметим, что $D-1$ независимых функций $U^{\mu}(\tau)$ (с учетом условия единичности $\left.U^{2}(\tau)=1\right)$, заданных на отрезке $[0,2 \pi]$, определяя движение струны, играют ту же роль, что и ее начальные положение и скорость в начально-краевой задаче [5].

\section{2. СТРУНА С БЕСКОНЕЧНО ТЯЖЕЛЫМ (ФИКСИРОВАННЫМ) КОНЦОМ}

Сконцентрируем внимание на исследовании релятивистской струны с конечной ненулевой массой $m_{1}$ на первом конце и с бесконечно тяжелым (фиксированным) вторым концом $\left(m_{2} \rightarrow \infty\right)$. Напомним, что такая модель описывает, в частности, и центрально-симметричные движения струны с одинаковыми массами на концах.

Как было показано выше, мировая поверхность такой струны однозначно определяется заданием единичного вектора скорости $U^{\mu}(\tau)$ первого конца на конечном отрезке (длина которого в случае параметризации (1.7) равна $2 \pi$ ), а также константы $W^{\mu}$ - скорости второго конца. Продолжение $U^{\mu}$ за пределы данного отрезка осуществляется с помощью интегрирования системы (1.21). Данную систему уравнений можно записать в эквивалентном виде, полученном комбинированием (1.19) и (1.20):

$$
U^{\prime \mu}(\tau+2 \pi)+Q(\tau+2 \pi) U^{\mu}(\tau+2 \pi)=\left(2 W^{\mu} W_{\nu}-\delta_{\nu}^{\mu}\right)\left[-U^{\prime \nu}(\tau)+Q(\tau) U^{\nu}(\tau)\right]
$$

где

$$
Q(\tau)=\sqrt{-U^{\prime 2}(\tau)}
$$


Эти нелинейные соотношения играют такую же роль, как и условия периодичности $\Psi_{+}^{\prime \mu}(\tau+2 \pi)=\Psi_{+}^{\prime \mu}(\tau)=\Psi_{-}^{\prime \mu}(\tau)$ (см. (1.15), [1]) для открытой струны с безмассовыми концами. В последнем случае периодические функции $\Psi_{ \pm}^{\prime \mu}(\tau)$ можно разложить в ряд Фурье, что служит исходным моментом для стандартной процедуры квантования $[1,8]$. В настоящей работе предпринимается попытка найти семейство независимых решений системы (2.1), которые выступали бы в той же роли, что и гармоники Фурье для периодических функций.

В рамках этого подхода вектор-функция $U^{\mu}(\tau)$, описываюшая движение струны с массивными концами, является аналогом функции $\Psi_{+}^{\prime \mu}(\tau)=\Psi_{-}^{\prime \mu}(\tau)$, определяющей мировую поверхность открытой свободной струны. Данная аналогия не случайна, именно изотропный вектор $\Psi_{ \pm}^{\prime \mu}(\tau)=\dot{X}^{\mu}(\tau, 0)$ играет роль $\mathbb{R}^{1, D-1}$-скорости для движущегося со скоростью света безмассового конца струны.

Будем считать, что вектор-функция $U^{\mu}(\tau)$ задана на отрезке $[0,2 \pi]$. Вследствие ее единичности

$$
U^{2}(\tau)=1
$$

число определенных на этом отрезке независимых функций, необходимых для полного описания мировых поверхностей рассматриваемой струны в $\mathbb{R}^{1, D-1}$, формально равно $D-1$. Однако из-за физической неразличимости "продольных колебаний", связанных с репараметризациями (1.9), число степеней свободы оказывается на единишу меньше. Это выражается, в частности, в том, что если $U^{\mu}(\tau)$ - решение системы $(2.1)-(2.2)$, то и вектор-функция $\tilde{U}^{\mu}(\tau)=U^{\mu}(\Theta(\tau))$, где

$$
\Theta(\tau)=\tau+\phi(\tau), \quad \phi(\tau+2 \pi)=\phi(\tau), \quad\left|\phi^{\prime}(\tau)\right|<1,
$$

также будет решением $(2.1)-(2.2)$. При этом

$$
Q(\tau) \longrightarrow \tilde{Q}(\tau)=\left[1+\phi^{\prime}(\tau)\right] Q(\Theta(\tau)) .
$$

Мировые поверхности, восстанавливаемые с помощью (1.20) по $U^{\mu}$ и $\tilde{U}^{\mu}$, совпадают, а функции $X^{\mu}(\tau, \sigma)$, их задаюшие, переходят друг в друга посредством репараметризации $(1.9)$. Осушествляюшую ее функцию $\phi(\tau)$, заданную на отрезке $[0,2 \pi]$, можно рассматривать как одну из $D-1$ функций на этом отрезке, определяюших движение струны. Следовательно, реальную физическую мировую поверхность определяют оставшиеся $D-2$ независимых функций, а $D-1$-ю мы можем выбрать по своему усмотрению.

Можно задать на отрезке $[0,2 \pi]$ функцию $Q(\tau)$ в качестве одной из независимых функций, определяюших вектор $U^{\mu}(\tau)$. При этом произвол в выборе $Q(\tau)(\tau \in[0,2 \pi])$ можно связать вследствие $(2.5)$ с "продольными колебаниями". Однако после фиксации $Q(\tau)$ выбор $D$ функций $U^{\mu}(\tau)$ на отрезке $[0,2 \pi]$ (а также и при произвольных $\tau$ ) будет ограничен двумя условиями: единичности $(2.3)$ и " $Q$-соответствия" (2.2). Число независимых функций по-прежнему равно $D-1$, роль одной из них берет на себя $Q(\tau)$.

Описанным образом может быть задано любое движение струны, рассматриваемой в этом разделе. Подчеркнем, что при $\tau>2 \pi$ функцию $Q(\tau)$ задавать произвольно нельзя - она однозначно определяется из системы (1.21).

Предположим, однако, что наряду с заданным на отрезке $[0,2 \pi]$ вектором $U^{\mu}$ нам известна функция $Q(\tau)$ при $\tau>2 \pi$. В этом случае можно, рассматривая формально (2.1) 
как линейную относительно $U^{\mu}$ систему обыкновенных дифференциальных уравнений, записать ее решение в следуюшем виде:

$$
U^{\mu}(\tau+2 \pi)=A_{\nu}^{\mu}\left\{-U^{\nu}(\tau)+e^{-T(\tau)} \int[Q(\tau)+Q(\tau+2 \pi)] U^{\nu}(\tau) e^{T(\tau)} d \tau\right\}
$$

Здесь введены обозначения $A_{\nu}^{\mu}=2 W^{\mu} W_{\nu}-\delta_{\nu}^{\mu}, T(\tau)=\int_{0}^{\tau+2 \pi} Q(\theta) d \theta$ и одно из слагаемых проинтегрировано по частям. Постоянная в неопределенном интеграле определяется из условия непрерывности $U^{\mu}$.

Представим вектор-функцию $U^{\mu}(\tau)$ произвольного физического движения струны в виде ряда

$$
U^{\mu}(\tau)=\sum_{n} u_{n}^{\mu}(\tau)
$$

где $u_{n}^{\mu}(\tau)$ - некоторые решения системы уравнений (2.1), соответствуюшие некоторому заданному $Q(\tau), \tau>0$. Вследствие “формальной линейности" (2.1) их сумма также будет решением этой системы. Но в отличие от их суммы $U^{\mu}(\tau)$ функции $u_{n}^{\mu}(\tau)$ по отдельности, вообще говоря, не удовлетворяют ни условию единичности $(2.3)$, ни условию соответствия заданному $Q(2.2)$, т.е. не задают физические мировые поверхности.

Первое из упомянутых условий, а именно $U^{2}=1$, можно интерпретировать как уравнение массовой поверхности, которому должен удовлетворять импульс $p^{\mu}=m_{1} U^{\mu}$ первого (подвижного) конца струны. Интересно отметить, что если изначально задать вектор-функцию $U^{\mu}(\tau)$ вне массовой поверхности $\left(U^{2} \neq 1\right)$, то система (1.13) по-прежнему единственным образом разрешима относительно $\Psi_{+}^{\prime \mu}$. Решение имеет сходный с $(1.20)$ вид

$$
\Psi_{ \pm}^{\prime \mu}(\tau)=\frac{1}{k_{1}}\left[ \pm U^{\prime \mu}(\tau)+Q(\tau) \cdot U^{\mu}(\tau)\right]
$$

Однако в роли $Q(\tau)$ здесь и соответственно в (2.1) будет выступать выражение $Q(\tau)=$ $-\frac{1}{2} \frac{d}{d \tau} \ln \left|U^{2}(\tau)-1\right|$.

Возвращаясь к разложению $(2.7)$, заметим, что в роли $u_{n}^{\mu}(\tau)$ может выступать всякая полная в классе $C_{[0,2 \pi]}$ система функций. В частности, можно разложить $U^{\mu}(\tau)$ на данном отрезке $[0,2 \pi]$ в ряд Фурье:

$$
U^{\mu}(\tau)=\sum_{n=-\infty}^{\infty} \alpha_{n}^{\mu} e^{-i n \tau}, \quad \tau \in[0,2 \pi], \quad \alpha_{-n}^{\mu}=\left(\alpha_{n}^{\mu}\right)^{*}
$$

(здесь отмечена вешественность $U^{\mu}$ ). Другой пример полной системы рассмотрен в разделе 3.

Необходимо отметить, что ряд (2.7) имеет вид (2.9) только на отрезке [0, $2 \pi]$. Функции $U^{\mu}(\tau)$ и $u_{n}^{\mu}(\tau)$, не будучи, вообше говоря, $2 \pi$-периодическими, продолжаются в область значений аргумента $\tau>2 \pi$ посредством решения системы $(2.1)-(2.2)$. Играюшая при этом важную роль величина $Q(\tau)$ определяется $U^{\mu}(\tau)$ - левой частью $(2.9)$ с помощью следуюшей процедуры. Для любой гладкой вектор-функции $U^{\mu}(\tau)$, заданной в виде (2.9) на отрезке $[0,2 \pi]$, сушествует единственное решение системы $(1.21)$, продолжаюшее $U^{\mu}$ при $\tau>2 \pi$. Подставим найденную таким образом $Q(\tau)$ в систему (2.1). Решая с помошью формулы (2.6) эту систему с $u_{n}^{\mu}$ в качестве неизвестных функций, мы можем 
продолжить на всю числовую полуось все “гармоники” $u_{n}^{\mu}(\tau)$, заданные на исходном отрезке в виде $(2.9)$.

Итак, всякое движение рассматриваемой здесь струны может быть представлено в виде суперпозищии (2.7), вообше говоря, нефизических состояний $u_{n}^{\mu}(\tau)$. Величины $\alpha_{n}^{\mu}$ однозначно определяют $u_{n}^{\mu}(\tau)$ вне зависимости от того факта, что не при всех $\tau$ функции $u_{n}^{\mu}$ имеют вид (2.9).

Линейная комбинация описываемых состояний также является состоянием, т.е. решением системы $(2.1)$ с данным $Q(\tau)$. В этом смысле $u_{n}^{\mu}$ можно назвать $Q$-состояниями. Эта их особенность связана с нелинейностью рассматриваемой струнной модели, которая полностью, естественно, не устранима. Нелинейность проявляется, в частности, в том, что если в заданном выражении (2.9) изменить только один из коэффициентов $\alpha_{n_{0}}^{\mu}$, то вследствие изменения $Q(\tau)$ при $\tau>0$ изменится система $(2.1)$ и все ее решения $u_{n}^{\mu}(\tau)$ при $\tau>2 \pi$ и с $n \neq n_{0}$. Впрочем, нелинейный в том же смысле характер имеет и следующее ниже условие (как и его аналог для свободной и замкнутой струн).

Заданная выражением (2.9) функция $U^{\mu}(\tau)$ определяет физическое движение струны тогда и только тогда, когда выполняется условие массовой поверхности (2.3). Достаточно потребовать его соблюдения на отрезке $[0,2 \pi]$, т.к. в этом случае решение системы (1.21) будет единичным при всех $\tau$ (домножив $(1.21)$ на $U^{\mu}$, получим $\frac{d}{d \tau} U^{2}(\tau+2 \pi)=0$ ).

Подставим ряд (2.9) в условие (2.3) и введем обозначение для амплитуд гармоник Фурье выражения $U^{2}(\tau)$ - аналогов операторов Вирасоро $[1,8]$ :

$$
L_{n}=\sum_{m=-\infty}^{\infty}\left(\alpha_{n-m} \alpha_{m}\right)
$$

Очевидно, что $L_{-n}=L_{n}^{*}$. В этих обозначениях условие массовой поверхности $(2.3)$ эквивалентно совокупности уравнений

$$
\begin{gathered}
L_{n}=0, \quad n=1,2 \ldots \\
L_{0} \stackrel{\text { def }}{=} \sum_{m=-\infty}^{\infty}\left(\alpha_{-m} \alpha_{m}\right)=\sum_{m=-\infty}^{\infty}\left|\alpha_{m}\right|^{2}=1 .
\end{gathered}
$$

Данные соотношения аналогичны тем, которые возникают как следствие разложения в ряд Фурье условий (1.6) в теории открытой свободной или замкнутой струны. При этом аналог условия $(2.12)$, а именно, $L_{0}=0$ приводит к уравнению массовой поверхности для струны $[1,8]$. В рассматриваемой здесь модели смысл данной процедуры проясняется: условие массовой поверхности (уравнение состояния) для струны (2.12) вытекает из условия массовой поверхности для массивного конца (2.3).

Отметим, что если взять за аксиомы соотношения (1.11) и (1.20), то ограничительные условия (1.6) и (2.3) оказываются эквивалентными:

$$
\Psi_{ \pm}^{\prime 2}(\tau)=0 \Longleftrightarrow U^{2}(\tau)=1
$$

Данное утверждение легко доказывается в обе стороны возведением в скалярный квадрат обеих частей соотношений соответственно (1.11) и (1.20). 
Ряд (2.7) вследствие (1.20) или (2.8) и свойств $Q$-состояний $u_{n}^{\mu}(\tau)$ приводит к разложению в ряд $\Psi_{ \pm}^{\mu}(\tau)$ и $X^{\mu}(\tau, \sigma): \Psi_{ \pm}^{\prime \mu}(\tau)=k_{1}^{-1} \sum\left[ \pm u_{n}^{\prime \mu}(\tau)+Q(\tau) \cdot u_{n}^{\mu}(\tau)\right]$, в частности,

$$
\Psi_{ \pm}^{\prime \mu}(\tau)=\frac{1}{k_{1}} \sum_{n=-\infty}^{\infty}[Q(\tau) \mp i n] \alpha_{n}^{\mu} e^{-i n \tau}, \quad \tau \in[0,2 \pi]
$$

Нелинейность задачи здесь явно присутствует в виде неразложенной функции $Q(\tau)$, которая должна удовлетворять следующим из (2.2) и (2.9) условиям:

$$
Q^{2}(\tau)=\sum_{n=-\infty}^{\infty}\left[\sum_{m=-\infty}^{\infty}(n-m) m\left(\alpha_{n-m} \alpha_{m}\right)\right] e^{-i n \tau}, \quad \tau \in[0,2 \pi] .
$$

Координаты точки струны $X^{\mu}(\tau, \sigma)$ выражаются через величины $\alpha_{n}^{\mu}$ с помощью $(2.13)$ и (1.5) в треугольнике на плоскости $\tau, \sigma$, ограниченном прямыми $\sigma=0, \tau=\sigma$ и $\tau+\sigma=2 \pi$.

Возможны иные способы представления $X^{\mu}(\tau, \sigma)$ с помощью ряда (2.7). Отметим лишь упомянутую выше возможность задания наряду с (2.9) произвольной функции $Q(\tau)$ на отрезке $[0,2 \pi]$ в виде аналогичного ряда Фурье $Q(\tau)=\sum q_{n} e^{-i n \tau}$. При этом возникает дополнительное условие $Q$-соответствия $(2.14)$ на данном отрезке.

Подобным же образом можно задавать значения $Q(\tau)$ на следуюших имеюших длину $2 \pi$ отрезках числовой оси. Это позволит записать на данном интервале явные решения (2.6) и добавит дополнительные связи.

Следует учесть, что значения $Q(\tau)$ и $Q(\tau+2 \pi)$ связаны некоторым соотношением, которое можно получить, домножив скалярно $(2.1)$ на $U^{\mu}(\tau+2 \pi)$ и $U^{\mu}(\tau)$. В первом случае с учетом $(2.3)$ и следуюшего из него соотношения $U^{\mu}(\tau) U_{\mu}^{\prime}(\tau)=0$ получим равенство

$$
Q(+)=-\left\langle U(+), U^{\prime}(\tau)\right\rangle+Q(\tau) \cdot G(\tau) .
$$

Здесь символ $(+)$ эквивалентен $(\tau+2 \pi)$, а также введены обозначения для билинейной операции в $\mathbb{R}^{1, D-1}$

$$
\langle f, g\rangle \stackrel{\text { def }}{=} f_{\mu}\left(2 W^{\mu} W_{\nu}-\delta_{\nu}^{\mu}\right) g^{\nu}
$$

и величины

$$
G(\tau)=\langle U(\tau+2 \pi), U(\tau)\rangle .
$$

“Домножая" в смысле $(2.16)$ на $U^{\mu}(\tau)$ обе части уравнения $(2.1)$, придем к следующему выражению: $\left\langle U^{\prime}(+), U(\tau)\right\rangle+Q(+) \cdot G(\tau)=Q(\tau)$

Складывая почленно последнее равенство с (2.15), получим после элементарных преобразований вышеупомянутое соотношение

$$
Q(\tau+2 \pi)-Q(\tau)=-\frac{d}{d \tau} \ln [1+G(\tau)]
$$

Если функция $Q(\tau)$ задана на отрезке $[2 \pi, 4 \pi]$, то наряду с возможностью получить явные продолжения "гармоник" $u_{n}^{\mu}$ на тот же отрезок мы приобретаем дополнительную связь. С учетом соотношения (2.18) можно записать ее и в виде $Q(+)$-соответствия $(2.2)$, и в виде $G$-соответствия $(2.17)(\tau \in[0,2 \pi])$. Оба эти условия следуют из требования $U^{2}(+)=1$, что легко проверить, возведя в квадрат обе части уравнения $(2.1)$. 
В заключительном разделе рассмотрим частньй случай задания $Q(\tau)$ в простейшем виде $Q=$ const на всей числовой оси.

\section{3. ДВИЖЕНИЯ С НАТУРАЛЬНОЙ ПАРАМЕТРИЗАЦИЕЙ ТРАЕКТОРИИ КОНЦА СТРУНЫ ( $Q=$ const)}

Сосредоточим внимание на движениях рассмотренной в предыдущем разделе релятивистской струны, которые характеризуются постоянным значением параметра (2.2):

$$
Q(\tau)=Q_{0}=\text { const }, \quad \tau \in \mathbb{R}, \quad Q_{0}>0, \quad 0<m_{1}<\infty, \quad m_{2} \rightarrow \infty .
$$

Сделаем предварительно несколько замечаний.

1. На мировой поверхности, связанной с данным движением, параметр $\tau$ пропорционален натуральному параметру $s$ (собственному времени) на траектории первого конца. Это следует из постоянства на траектории кривизны $k_{1}=\gamma / m_{1}$, которую можно найти как модуль вектора кривизны $k_{1} \nu^{\mu}=d U^{\mu} / d s$, что приводит к соотношению $Q(\tau)|d \tau / d s|=k_{1}$.

2. Движения струны с $2 \pi$-периодическим $Q(\tau)>0$ также сводятся к виду $(3.1)$, т.к. с помощью замены параметра (2.4), связанной с “продольными колебаниями” и сохраняюшей мировую поверхность, всегда можно, учитывая (2.5), добиться постоянства $Q$ в новых координатах.

3. Для движений струны, сводимых к виду (3.1), вследствие (2.18) постоянна величина $G(\tau)$.

4. Для произвольного движения струны с помощью репараметризации (2.4) достичь постоянства $Q$ при $\tau \in \mathbb{R}$, вообше говоря, невозможно. Более того, движения с $2 \pi$-периодическим $Q$, сводимые к виду (3.1), образуют множество меры нуль в классе всех мировых поверхностей рассматриваемой струны. Дело в том, что, реализовав произвол $(2.4)$ или (1.9), связанный с периодической функцией $\phi(\tau)$, мы всегда можем добиться постоянства $Q(\tau)$ только на отрезке длиной $2 \pi$, в частности на $[0,2 \pi]$. Попытка наложить дополнительное условие $Q=$ const при $\tau \in[2 \pi, 4 \pi]$ приводит к ограничению на выбор начальной функции $U^{\mu}(\tau), \tau \in[0,2 \pi]$. Два условия постоянства $Q$ на двух смежных отрезках в совокупности с условием (2.3) сужают выбор порождающих движения струны $U^{\mu}$ до $D-3$ произвольных функций на отрезке $[0,2 \pi]$. В частности, в плоском случае $(D=3)$ условие $(3.1)$ фиксирует мировую поверхность струны с точностью до движений (более строгое обоснование дано ниже).

Последнее замечание отражает ограниченность класса мировых поверхностей струны вида (1.1) с натуральным параметром $\tau$ на траекториях концов струны (в рамках условий (1.2)). Попытка квантового описания такой системы впервые была сделана в [10] (см. также [1]). Был использован тот факт, что требование натуральности параметра линеаризует краевые условия (1.4). С учетом того, что при $\tau=\left.s \quad \dot{X}^{2}\right|_{\sigma=\sigma_{a}}=1$, эти условия примут вид

$$
\left.\left[\ddot{X}^{\mu}+(-1)^{a} k_{a} X^{\prime \mu}\right]\right|_{\sigma=\sigma_{a}}=0, \quad a=1,2, \quad k_{a}=\gamma / m_{a} .
$$

Решения задачи (1.3),(3.2) были найдены в виде ряда по собственным функциям, характеризуемым частотами - корнями трансцендентного уравнения $[1,4,10]$. Однако наложение условий (1.2) существенно ограничивает класс таких движений по причине, связанной с замечанием 4. 
Условие (3.1) линеаризует как выражение (1.4), так и систему (2.1). Поэтому можно решить соответствуюшую задачу для струны с $m_{2} \rightarrow \infty$, исследовав решения системы (2.1) с $Q(\tau)=Q_{0}$, имеющие вид $u^{\mu}(\tau)=\alpha^{\mu} e^{-i \omega \tau}, \tau \in \mathbb{R}$.

Подстановка этого выражения в $(2.1),(3.1)$ приводит к соотношению

$$
\left(2 W^{\mu} W_{\nu}-\delta_{\nu}^{\mu}\right) \alpha^{\nu}=\frac{Q_{0}-i \omega}{Q_{0}+i \omega} e^{-2 \pi i \omega} \alpha^{\mu} \equiv \lambda(\omega) \alpha^{\mu}
$$

которое означает, что $\alpha^{\mu}$ должен быть собственным вектором матрищы $A_{\nu}^{\mu}=2 W^{\mu} W_{\nu}-$ $\delta_{\nu}^{\mu}$. Эта матрица имеет два собственных значения: $\lambda=1$, которому отвечает собственньй вектор $W^{\mu}$, и $\lambda=-1$, которому соответствует $D-1$ линейно независимых собственных векторов $\beta^{\mu}$ ортогональных $W^{\mu}:(W \beta)=0$. Различие собственных значений приводит к различию частот $\omega$ для $\alpha^{\mu}$, лежаших в линейной оболочке $W^{\mu}$ и в ее ортогональном дополнении. Обозначив эти частоты соответственно $\Omega$ и $\omega$, выпишем уравнения $\lambda(\Omega)=1$ и $\lambda(\omega)=-1$, которым они удовлетворяют:

$$
\frac{\Omega}{Q_{0}}=-\operatorname{tg} \pi \Omega, \quad \frac{\omega}{Q_{0}}=\operatorname{ctg} \pi \omega
$$

Каждое из этих трансцендентных уравнений имеет счетный набор корней $\Omega_{n}$ и $\omega_{n}$, расположенных симметрично относительно начала координат. Произвольная линейная комбинация гармоник с частотами $\Omega_{n}$ и $\omega_{n}$ и соответствуюшими собственными векторами

$$
U^{\mu}(\tau)=\sum_{n=-\infty}^{\infty} u_{n}^{\mu}(\tau)=\sum_{n=-\infty}^{\infty}\left(\alpha_{n} W^{\mu} e^{-i \Omega_{n} \tau}+\beta_{n}^{\mu} e^{-i \omega_{n} \tau}\right), \quad\left(W \beta_{n}\right)=0
$$

является решением (2.1) с $Q=Q_{0}$. Однако сходяшийся ряд (3.4) порождает физическое движение струны только в случае вешественности $U^{\mu}$, т.е. при $\alpha_{-n}=\left(\alpha_{n}\right)^{*}$ и $\beta_{-n}^{\mu}=$ $\left(\beta_{n}^{\mu}\right)^{*}$, и при выполнении условий массовой поверхности $(2.3)$ и $Q_{0}$-соответствия $(2.2)$.

При возведении ряда (3.4) в квадрат мы получим бесконечный набор гармоник с частотами $\Omega_{m}+\Omega_{n}$ и $\omega_{m}+\omega_{n}$. Строгая монотонность изменения расстояний между соседними положительными корнями уравнений (3.3) приводит к тому, что упомянутые суммы, если только $m+n \neq 0$, не могут быть представлены в виде сумм двух других частот. Следовательно, все слагаемые с отмеченной суммой частот в показателях должны быть равны нулю, т.к. остальные гармоники не могут в точности компенсировать их вклад на всей числовой оси. Предположив противное, мы придем к равенству $0=\sum \rho_{n} e^{-i \chi_{n} \tau}$, где не все $\rho_{n}$ равны нулю, а все $\chi_{n}$ различны. Преобразование Фурье обеих частей этого равенства показывает его невозможность.

В отношении амплитуд $\alpha_{n}$ при времениподобном векторе $W^{\mu}$ ограничение $(2.3)$ и изложенные соображения приводят к тому, что все $\alpha_{n}$ равны нулю за исключением $\alpha_{0}$ $\left(\alpha_{0}>0\right)$, которому отвечает частота $\Omega_{0}=0$.

Следуюшее из $(2.3)$ условие $\left(\beta_{m} \beta_{n}\right) e^{-i\left(\omega_{m}-\omega_{n}\right) \tau}=0(m \neq n)$ есть требование взаимной ортогональности всех векторов $\beta_{n}^{\mu}$ и $\beta_{n}^{\mu *}, n \geqslant 1$ (здесь скалярное произведение: $\left.(\alpha \beta)=\alpha^{\mu} \beta_{\mu}^{*}\right)$. Нетрудно показать, что в $\mathbb{R}^{1, D-1}$ существует не более $D-1$ взаимно ортогональных векторов $\beta_{n}^{\mu}$ и $\beta_{n}^{\mu *}$ таких, что $\left(W \beta_{n}\right)=0$. 
Итак, всякий ряд (3.4) конечен

$$
U^{\mu}(\tau)=\alpha_{0} W^{\mu}+2 \sum_{j=1}^{[(D-1) / 2]} \operatorname{Re} \beta_{n_{j}}^{\mu} e^{-i \omega_{n_{j}} \tau}, \quad\left(W \beta_{n_{j}}\right)=0, \quad\left(\beta_{n_{i}} \beta_{n_{j}}\right)=0
$$

и число различных частот $\omega_{n}$ в нем равно целой части $(D-1) / 2$. В частности, для $D=4$ и для плоского случая $(D=3)$ возможно движение только с одной частотой $\omega_{n}$.

Используя ортогональность $\alpha_{0} W^{\mu}$ и $\beta_{n}^{\mu}$, мы всегда можем наложить на амплитуды этих векторов условия массовой поверхности $(2.3)$ и $Q_{0}$-соответствия $(2.2)$. Если вместо $\beta_{n}^{\mu}, \beta_{n}^{\mu *}$ ввести пару ортогональных вешественных векторов $b_{n} e_{n}^{\mu}=2 \operatorname{Re} \beta_{n}^{\mu}$ и $b_{n} g_{n}^{\mu}=$ $2 \operatorname{Im} \beta_{n}^{\mu},\left(e_{n} g_{n}\right)=0, e_{n}^{2}=g_{n}^{2}=-1$, то эти условия соответственно примут вид

$$
\begin{aligned}
U^{2}=1 & \Longrightarrow \alpha_{0}^{2}-\left(b_{n_{1}}^{2}+\cdots+b_{n_{N}}^{2}\right)=1, \quad N=[(D-1) / 2], \\
U^{\prime 2}=-Q_{0}^{2} & \Longrightarrow\left(\omega_{n_{1}}^{2} b_{n_{1}}^{2}+\cdots+\omega_{n_{N}}^{2} b_{n_{N}}^{2}\right)=Q_{0}^{2} .
\end{aligned}
$$

Покажем, что в пространствах Минковского размерностей $D=3$ или 4 имеет место нетривиальный факт, а именно, в них возможно только одно движение струны без особенностей на мировой поверхности, удовлетворяющее условию (3.1). Оно определяется минимальной частотой $\omega_{1}>0$, причем величины $b_{1}$ и $\alpha_{0}$ с помощью $(3.6),(3.7)$ выражаются через $Q_{0}$ :

$$
U^{\mu}(\tau)=\omega_{1}^{-1}\left[\sqrt{Q_{0}^{2}+\omega_{1}^{2}} W^{\mu}+Q_{0}\left(e_{1}^{\mu} \cos \omega_{1} \tau+g_{1}^{\mu} \sin \omega_{1} \tau\right)\right] .
$$

Определенная с помощью (1.20) и (1.5) мировая поверхность этой струны

$$
X^{\mu}(\tau, \sigma)=\frac{Q_{0} \sqrt{Q_{0}^{2}+\omega_{1}^{2}}}{k_{1} \omega_{1}^{2}}\left\{W^{\mu} \omega_{1} \tau+\cos \left(\omega_{1} \sigma+\varphi_{1}\right)\left[e_{1}^{\mu} \sin \omega_{1} \tau-g_{1}^{\mu} \cos \omega_{1} \tau\right]\right\}
$$

(здесь $\left.\varphi_{1}=\operatorname{arctg} \frac{\omega_{1}}{Q_{0}}\right)$ представляет собой хорошо известный геликоид, заметаемый равномерно врашающейся струной, которая сохраняет форму отрезка прямой $[2,3,6]$.

Мировые поверхности вида (3.8) с частотами $\omega_{n}, n \geqslant 2$, будут иметь особенности при $\sigma \in[0, \pi]$ сушествует $n-1$ линия $\sigma=\mathrm{const}\left(\right.$ нули функции $\left.\sin \left(\omega_{n} \sigma+\varphi_{n}\right)\right)$, на которых величина $X^{\prime \mu}$ обрашается в нуль, что влечет за собой обрашение в нуль индуцированной метрики $\left(\dot{X}^{2}=0\right)$. Соответствуюшие точки движутся со световой скоростью. Физически это можно воспринимать как врашающуюся струну, сложенную $n$ раз, или как $n$ совмещенных в пространстве струн, участвующих в едином вращении. При этом коншы струн, совпадающие с изломами, безмассовы; первая из струн имеет массы на концах $m_{1}$ и 0, последняя -0 и $\infty$.

Интересно отметить, что этих особенностей $\left(\dot{X}^{2}=0\right)$ лишены движения струны в пространствах размерности $D \geqslant 5$, определяемые выражением (3.5) и содержашие $N=$ 2 и более различных частот $\omega_{n}$, например

$$
U^{\mu}(\tau)=\sqrt{1+b_{1}^{2}+b_{2}^{2}} W^{\mu}+b_{1}\left(e_{1}^{\mu} \cos \omega_{1} \tau+g_{1}^{\mu} \sin \omega_{1} \tau\right)+b_{2}\left(e_{2}^{\mu} \cos \omega_{2} \tau+g_{2}^{\mu} \sin \omega_{2} \tau\right)
$$

Множество этих мировых поверхностей описывается $N$ параметрами, в качестве которых можно взять $Q_{0}$ и $b_{n_{i}} / b_{n_{j}}$.

4 Теоретическая и математическая физика, т. 107, № 1, 1996 г. 


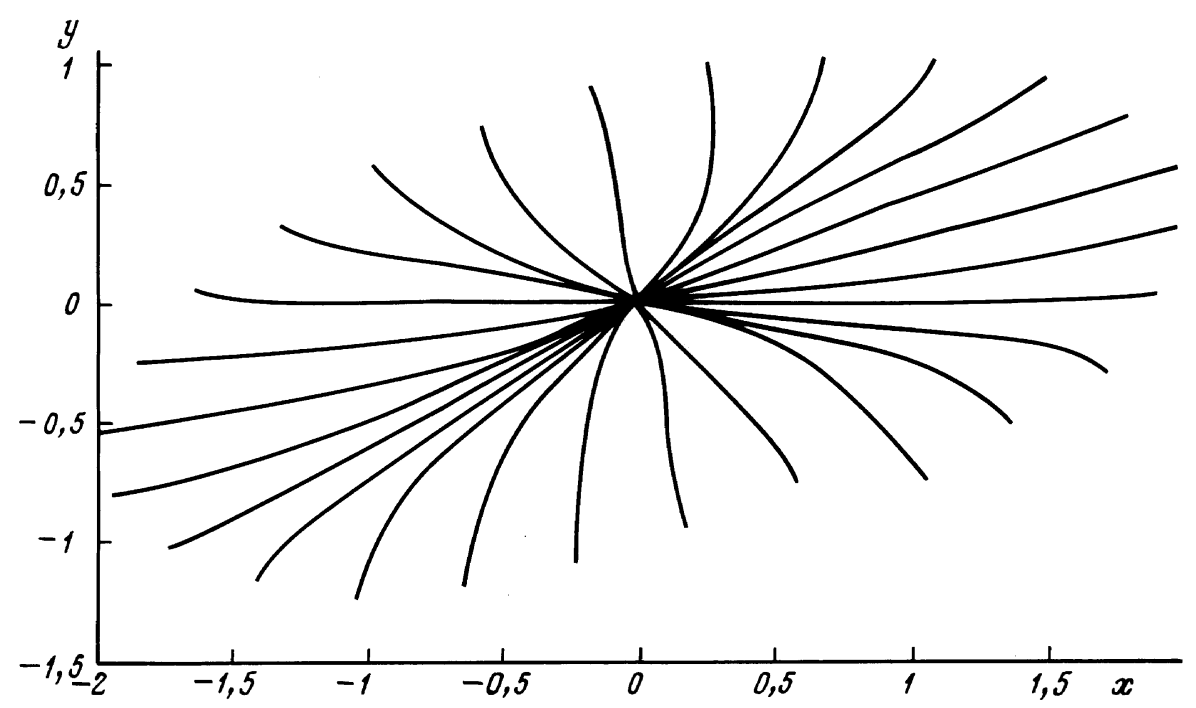

Семейства функций $e^{-i \Omega_{n} \tau}$ и $e^{-i \omega_{n} \tau}$ полны в классе $C_{[0,2 \pi]}$, т.е. любую гладкую функцию $U^{\mu}(\tau)$, заданную на $[0,2 \pi]$, можно на этом отрезке разложить в ряд (3.4). Но тот же вид за пределами этого отрезка имеют только функции, представимые конечной суммой (3.5). Следовательно, приведенная классификация охватываемых условием (3.1) движений струны полна, другими словами, всякое движение струны с $m_{2} \rightarrow \infty$, допускающее натуральную параметризацию, описывается суммой (3.5).

Строгое доказательство этого утверждения будет представлено в другой работе, здесь же ограничимся лишь его схемой.

Приведенные выше результаты могут быть получены с помощью метода Фурье, приложенного к задаче (1.3), (3.2) [1-10]. Равенство $k_{2}=0$ в (3.2) преобразует, например, для пространственных координат (в некоторой системе отсчета) это краевое условие к виду $\left.X^{\mu}\right|_{\sigma=\pi}=0$. Подстановка выражения $X^{\mu}(\tau, \sigma)=e^{\mu} f(\sigma) e^{-i \omega \tau}$ в (1.3), (3.2) приводит для этих координат к несколько обобщенной задаче Штурма-Лиувилля

$$
f^{\prime \prime}+\omega^{2} f=0, \quad \omega^{2} f(0)+k_{1} f^{\prime}(0)=0, \quad f(\pi)=0
$$

собственные значения которой $\omega_{n}$ определяются вторым уравнением (3.3) (т.к. $\tau=s$, то $\left.k_{1}=Q_{0}\right)$, собственные функции имеют вид $f_{n}=\cos \omega_{n} \sigma-\left(\omega_{n} / Q_{0}\right) \sin \omega_{n} \sigma$. Они образуют полную ортогональную систему в $C_{[0, \pi]}[10-11]$. Пополнив ее функциями $\tilde{f}_{n}=$ $f_{n}^{\prime}(\sigma)$, мы получим эквивалентную $e^{-i \omega_{n} \tau}$ систему, полную на отрезке длиной $2 \pi$.

Полнота системы в (3.4) позволяет использовать ее, отождествляя (3.4) с рядом (2.7). Но для произвольного не сводящегося к (3.5) движения функции $u_{n}^{\mu}(\tau)$ имеют вид (3.4) только на отрезке $[0,2 \pi]$. За пределы этого отрезка они продолжаются с помощью описанной в разделе 2 процедуры.

\section{ЗАКЛЮЧЕНИЕ}

Модель струны с фиксированным концом была помешена в центр внимания в данной работе ради простоты изложения предлагаемого подхода, которьй включает в себя разложение описьвающей движение струны функции $U^{\mu}(\tau)$ в ряд (2.7). Переход к случаю 
$m_{2}=0$ сводится к замене матрицы $A_{\nu}^{\mu}=2 W^{\mu} W_{\nu}-\delta_{\nu}^{\mu}$ на $\delta_{\nu}^{\mu}$. Минимальных усилий требует и обобшение на случай $0<m_{2}<\infty$. Отсутствие при любых $m_{2}$ явных выражений для $U^{\mu}(\tau)$ и $u_{n}^{\mu}(\tau)$ при $\tau>2 \pi$ не должно вызывать сомнений в их существовании и единственности.

Отметим, что процедура численного определения этих функций и порождаемых ими мировых поверхностей довольно проста.

В качестве иллюстрации на рисунке показано движение струны в $\mathbb{R}^{1,2}$ (ее положения через равные промежутки времени $\left.\Delta t=0,5, t \equiv X^{0}\right)$, полученное численным решением системы (1.21) со следующими исходными данными на отрезке $[0,2 \pi]$ : $U^{\mu}(\tau)=\left\{\left(1+f^{2}\right)^{1 / 2} ; f(\tau) \cos 0,2 \tau ; f(\tau) \sin 0,2 \tau\right\}, f(\tau)=2-0,2 \tau, k_{1}=1$.

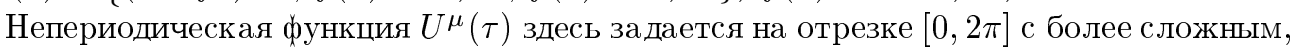
чем для $2 \pi$-периодической функции, правилом продолжения (1.21). Возможно, эта сложность неизбежно следует из неустранимой нелинейности задачи, в которой попытка линеаризации, предпринятая в разделе 3 , приводит к существенному сужению класса рассматриваемых движений, в частности, при $D \leqslant 4$ - фактически к единственному движению (3.8).

Автор глубоко признателен Б. М. Барбашову, В. В. Нестеренко и А. М. Червякову за основательное обсуждение работы на семинаре.

\section{Список литературы}

[1] Barbashov B. M., Nesterenko V. V. Introduction to the relativistic string theory. Singapore: World scientific, 1990; Барбашов Б. М., Нестеренко В. В. Модель релятивистской струны в физике адронов. М.: Энергоатомиздат, 1987.

[2] Chodos A., Thom C. B. // Nucl. Phys. 1974. V. B72. № 3. P. 509-522.

[3] Барбашов Б. М., Червяков А. М. // ТМФ. 1988. V. 74. №3. Р. 430-439; 1991. V. 89. № 1. P. $105-120$.

[4] Barbashov B. M. // Nucl. Phys. 1977. V. B129. №1. P. 175-188.

[5] Барбашов Б. М., Шаров Г. С. // ТМФ. 1994. Т. 101. № 2. С. 253-271.

[6] Barbashov B. M. Classical dynamics of rotating relativistic string with massive ends: the Regge trajectories and quark masses: Preprint E2-94-444. Dubna: JINR, 1994.

[7] Морозов А. Ю. // УФН. 1992. Т. 162. № 8. С. 83-176.

[8] Грин М., Швари, Дж., Виттен Э. Теория суперструн. Т. 1. М.: Мир, 1990.

[9] ШШаров Г. С. // ТМФ. 1995. Т. 102. № 1. С. 150-159.

[10] Барбашов Б. М., Нестеренко В. В. // ТМФ. 1977. Т. 31. № 3. С. 291-299.

[11] Стеклов В. А. Основные задачи математической физики. М.: Наука, 1983.

Тверской государственный университет

Поступила в редакцию 31.VII.1995 г.

\section{G.S. Sharov}

\section{ANALOGS OF FOURIER SERIES FOR A RELATIVISTIC STRING MODEL WITH MASSIVE ENDS}

A description of motions of a relativistic string with massive ends (for the model with a finite mass on the first end and infinite one on the second end) is proposed. This approach uses the expansion of the string world surface in a series that doesn't reduce to the usual Fourier series due to nonlinearity of the problem. A state equation of the string resultes from the mass shell condition of its end. World surfaces of this string, on which linearization of a boundary condition due to natural parametrization of moving end trajectory is possible, are classified. The set of such world surfaces is shown to be limited in particular in 2+1- and 3+1-dimensional Minkowski spaces all of them reduce to the helicoid. 